\title{
SOFT PHOTON PRODUCTION RATE IN RESUMMED PERTURBATION THEORY OF HIGH TEMPERATURE QCD
}

\author{
R. Baier ${ }^{1}$, S. Peigné ${ }^{2}$ and D. Schiff ${ }^{2}$ \\ ${ }^{1}$ Fakultät für Physik, Universität Bielefeld, D-33501 Bielefeld, Germany \\ ${ }^{2}$ LPTHE ${ }^{\dagger}$, Université Paris-Sud, Bâtiment 211, F-91405 Orsay, France
}

\begin{abstract}
We calculate the production rate of soft real photons from a hot quark - gluon plasma using Braaten - Pisarski's perturbative resummation method. To leading order in the QCD coupling constant $g$ we find a logarithmically divergent result for photon energies of order $g T$, where $T$ is the plasma temperature. This divergent behaviour is due to unscreened mass singularities in the effective hard thermal loop vertices in the case of a massless external photon.
\end{abstract}

LPTHE-Orsay 93/46

BI-TP 93/55

November 1993

\footnotetext{
† Laboratoire associé du Centre National de la Recherche Scientifique
} 


\section{INTRODUCTION}

Theoretical investigations predict the formation of a quark - gluon plasma (QGP) in high-energy heavy ion collisions. Many signatures for this new phase of nuclear matter have been proposed, in particular electromagnetic ones : photon production [1] by the QGP is expected to be an interesting signal, as the mean free path of the photon $\gamma$ in the thermal medium is expected to be larger than the size of the plasma, at least when the energy of the $\gamma$ is not too small.

The present paper is concerned with real direct photon production, assuming that the photon is not thermalized. The production rate of hard photons (with energy $E \sim T$, where $T$ is the plasma temperature) has already been studied in great detail [1 - 7]. Especially when applying the framework of the resummed perturbative expansion of Braaten and Pisarski [8 - 11], it has been demonstrated that mass singularities due to the exchange of massless quarks are shielded by effects due to Landau damping [5 - 6]. In the following we are dealing with the soft photon production rate $(E \sim g T$, where $g$ is the QCD coupling constant), whose calculation requires not only to use resummed quark propagators but also dressed vertices. This should allow us to extract the (finite) leading contribution to the production rate, thus completing the list of predictions of electromagnetic signals: real and virtual [12 - 14] photon rates.

The main result of the present work is that contrary to the hard photon case, for soft real photons the resummation advocated by Braaten and Pisarski does not succeed to screen mass singularities, i.e. in this case the resummed perturbative expansion fails to give a finite contribution at leading order for a physical quantity.

The outline of the paper is the following : in section 2, we briefly review the situation

for hard photon production. In section 3, we deal with soft photons, showing the origin of mass singular terms and exhibiting them, then extracting the singular contribution using dimensional regularisation. Section 4 is devoted to a short discussion.

\section{HARD PHOTON PRODUCTION RATE}

The Born calculation of the hard photon production rate, which uses a bare internal quark propagator for the first order annihilation and Compton scattering amplitudes, gives as the leading term [2-7] in the limit of vanishing bare quark mass $m$ : 


$$
E \frac{d W}{d^{3} \vec{p}} \simeq \frac{e_{q}^{2} \alpha \alpha_{s}}{2 \pi^{2}} T^{2} e^{-E / T} \ln \left(\frac{E T}{m^{2}}\right)
$$

where $E>T$. $\alpha$ is the fine structure constant, $e_{q}$ the quark charge and $\alpha_{s}=g^{2} / 4 \pi$.

The obvious problem with eq. (1) is that the photon production rate is divergent when $m$ tends to zero. Thus the leading order Born calculation is not satisfactory.

Indeed, when the momentum transfer of the exchanged quark, e.g. in the Compton process, is soft of $O(g T)$ one has to resum an infinite set of diagrams contributing to the same order as the Born term. This resummation program has been proposed by Braaten and Pisarski $[8$ - 11]. In case of the propagator it amounts to replace the bare by an effective one, whenever the momentum is soft.

The main characteristic property of the effective propagator is that it is dynamically screened on the momentum scale of order $g T$ for space-like momenta, due to the mechanism of Landau damping. The soft scale is characterized by the fermion mass induced by temperature, i.e. by $m_{f}=\sqrt{2 \pi \alpha_{s} / 3} T$. It acts as an infra-red cut-off, and in eq. (1) one may replace $m$ by $m_{f}$ [3]. This heuristic manipulation yields in fact the right result in the leading-logarithm approximation. The rigorous calculation $[5-6]$, which also allows to find the numerical coefficient appearing inside the log, leads to:

$$
E \frac{d W}{d^{3} \vec{p}} \simeq \frac{e_{q}^{2} \alpha \alpha_{s}}{2 \pi^{2}} T^{2} e^{-E / T} \ln \left(\frac{c}{\alpha_{s}} \frac{E}{T}\right)
$$

with $c \simeq 0.23$, when $E>T$.

One obtains a finite production rate for hard photons. The $\ell n\left(\frac{1}{\alpha_{s}}\right)$ dependence in eq. (2) is a reminiscence of the logarithmic divergence of the Born term, which indeed becomes dynamically screened after resummation.

In the following we want to know whether the resummed perturbative expansion achieves the same screening of mass singularities in the case of soft photon production, i.e. for photon energies of $O(g T)$.

\section{SOFT PHOTON PRODUCTION RATE}

The production rate may be computed in a systematic way by evaluating the imaginary part of the photon polarization tensor: 


$$
E \frac{d W}{d^{3} \vec{p}}=-\frac{1}{(2 \pi)^{3}} n_{B}(E) \operatorname{Im} \Pi_{\mu}^{\mu}(E, \vec{p})
$$

where $\Pi_{\mu}^{\mu}$ is first calculated in the euclidean formalism*. The Bose - Einstein distribution is denoted by $n_{B}$.

When the photon energy $E$ is of order $g T$, either $k$ and $k^{\prime}$ (Fig. 1) are soft $(\sim g T)$, and both quark propagators have to be resummed, or $k$ and $k^{\prime}$ are hard $(\sim T)$, but the latter contribution is suppressed by a factor $g^{2}$ and we shall neglect it. By evaluating the soft photon production rate according to eq. (3) we thus consider both internal quark propagators as soft ones. As the photon momentum $P=k+k^{\prime}$ is soft, vertices have also to be resummed and the relevant photon polarization tensor entering eq. (3) is shown in Fig. 1.

As the internal quark propagators are resummed, we expect that screening occurs as for the hard photons, and no divergence appears when $k$ or $k^{\prime}$ are vanishing. However, the introduction of effective vertices, though necessary to take into account all diagrams contributing to the rate at leading order in $g$, will be shown to lead to unscreened collinear divergences.

\subsection{Resummed photon self energy}

In order to evaluate the production rate (eq. (3)) we first consider $\Pi_{\mu}^{\mu}$ :

$$
\Pi_{\mu}^{\mu}(E, \vec{p})=e_{q}^{2} e^{2} N_{c} T \sum_{k_{4}} \int \frac{d^{3} \vec{k}}{(2 \pi)^{3}} 3 \operatorname{tr}\left[^{*} \Delta(k)^{*} \Gamma^{\mu}\left(k, k^{\prime} ;-P\right)^{*} \Delta\left(-k^{\prime}\right)^{*} \Gamma_{\mu}\left(-k^{\prime},-k ; P\right)\right]
$$

where $N_{c}$ is the number of colours and ${ }^{*} \Delta(k)$ is the effective quark propagator :

$$
{ }^{*} \Delta(k)=\frac{1}{2}\left(\frac{\gamma \cdot k_{+}}{D_{+}(k)}+\frac{\gamma \cdot k_{-}}{D_{-}(k)}\right)
$$

with

$$
k_{ \pm}=(1, \pm i \widehat{k}) \quad, \quad \widehat{k}=\vec{k} /|\vec{k}|
$$

\footnotetext{
$* \Pi_{\mu}^{\mu}$ is evaluated for $p_{4}=2 \pi n T$ and then continued according to $i p_{4} \rightarrow E$. In the imaginary time formalism, the euclidean Dirac algebra $\left\{\gamma^{\mu}, \gamma^{\nu}\right\}=2 \delta^{\mu \nu}$ is used.
} 
The functions $D_{ \pm}(k)$ are given in [13 - 17]. The effective quark - photon vertex [10 - 11, 13 - 14] is represented by:

$$
\begin{gathered}
{ }^{*} \Gamma^{\mu}=\gamma^{\mu}+m_{f}^{2} \int \frac{d \Omega}{4 \pi} \frac{Q^{\mu} Q}{(Q k)\left(Q k^{\prime}\right)} \\
Q=(i, \widehat{Q})
\end{gathered}
$$

The second term in the r.h.s. of eq. (6) is the hard thermal loop correction - in terms of an angular integral - to the bare vertex $\gamma^{\mu}$. $Q$ is a light-like vector, $Q^{2}=0$; the inner product $Q \cdot k=Q_{4} k_{4}+\widehat{Q} \cdot \vec{k}$ is denoted by $(Q k)$.

The Dirac trace in eq. (4) is split into three terms according to the number of hard loop corrections, cf. eq. (6): they are denoted by $\operatorname{tr}(0), \operatorname{tr}(1), \operatorname{tr}(2)$, respectively. We get :

$$
\Pi_{\mu}^{\mu}=e_{q}^{2} e^{2} N_{c} T \sum_{k_{4}} \int \frac{d^{3} \vec{k}}{(2 \pi)^{3}}[\operatorname{tr}(0)+\operatorname{tr}(1)+\operatorname{tr}(2)]
$$

where

$$
\begin{gathered}
\operatorname{tr}(0)=2 \sum_{i, j= \pm} \frac{\left(k_{i} k_{j}^{\prime}\right)}{D_{i} D_{j}^{\prime}} \\
\operatorname{tr}(1)=-4 m_{f}^{2} \int \frac{d \Omega}{4 \pi} \frac{1}{(k Q)\left(k^{\prime} Q\right)} \sum_{i, j= \pm} \frac{\left(k_{i} Q\right)\left(k_{j}^{\prime} Q\right)}{D_{i} D_{j}^{\prime}}, \\
\operatorname{tr}(2)=-m_{f}^{4} \int \frac{d \Omega_{1}}{4 \pi} \int \frac{d \Omega_{2}}{4 \pi} \frac{\left(Q_{1} Q_{2}\right)}{\left(k Q_{1}\right)\left(k Q_{2}\right)\left(k^{\prime} Q_{1}\right)\left(k^{\prime} Q_{2}\right)} \\
\sum_{i, j= \pm} \frac{1}{D_{i} D_{j}^{\prime}}\left[\left(Q_{1} k_{i}\right)\left(Q_{2} k_{j}^{\prime}\right)+\left(Q_{1} k_{j}^{\prime}\right)\left(Q_{2} k_{i}\right)-\left(Q_{1} Q_{2}\right)\left(k_{i} k_{j}^{\prime}\right)\right] .
\end{gathered}
$$

The primed quantities depend on the momentum $k^{\prime}=P-k$.

To obtain the imaginary part of $\Pi_{\mu}^{\mu}$ we use the identity [13 - 14] :

$$
\begin{gathered}
\operatorname{Im} T \sum_{k_{4}=2 \pi n T} f\left(i k_{4}\right) f^{\prime}\left(i\left(p_{4}-k_{4}\right)\right)= \\
\pi\left(1-e^{E / T}\right) \int_{-\infty}^{\infty} d \omega \int_{-\infty}^{\infty} d \omega^{\prime} n_{F}(\omega) n_{F}\left(\omega^{\prime}\right) \delta\left(E-\omega-\omega^{\prime}\right) \rho(\omega) \rho^{\prime}\left(\omega^{\prime}\right)
\end{gathered}
$$


where $n_{F}$ is the Fermi - Dirac distribution, and $\rho, \rho^{\prime}$ are the spectral densities associated with $f, f^{\prime}$, respectively:

$$
\rho(\omega)=\lim _{\varepsilon \rightarrow 0} \frac{1}{\pi} \operatorname{Im} f(\omega+i \varepsilon)
$$

Eq. (8) can be used only if the dependence of $\Pi_{\mu}^{\mu}$ on $i k_{4}$ and $i k_{4}^{\prime}$ is factorized. Thus, as proposed by Wong [14], it is convenient to take the discontinuity of $\Pi_{\mu}^{\mu}$ in the factorized form (7), before integrating over $d \Omega$.

When the continuation $i k_{4} \rightarrow \omega+i \varepsilon$ is performed, the functions with non-vanishing discontinuities are $\left[D_{ \pm}(k)\right]^{-1},\left[(Q k) D_{ \pm}(k)\right]^{-1},\left[\left(Q_{1} k\right)\left(Q_{2} k\right) D_{ \pm}(k)\right]^{-1}$ appearing in the terms $\operatorname{tr}(0), \operatorname{tr}(1), \operatorname{tr}(2)$ of eq. (7). The associated spectral densities are denoted by $\rho_{ \pm}$, $\sigma_{ \pm}, \tau_{ \pm}$, respectively. For space-like momentum $k, \omega<|\vec{k}|$, they read:

$$
\begin{gathered}
\rho_{ \pm}=\frac{1}{\pi} \operatorname{Im}\left[D_{ \pm}\right]^{-1}=\beta_{ \pm}(\omega,|\vec{k}|) \\
\sigma_{ \pm}=\frac{1}{\pi} \operatorname{Im}\left[(Q k) D_{ \pm}\right]^{-1}=\mathbf{P}\left(\frac{1}{Q k}\right) \beta_{ \pm}-\delta(Q k) \alpha_{ \pm} \\
\tau_{ \pm}=\frac{1}{\pi} \operatorname{Im}\left[\left(Q_{1} k\right)\left(Q_{2} k\right) D_{ \pm}\right]^{-1}=\left[\mathbf{P}\left(\frac{1}{Q_{1} k}\right) \mathbf{P}\left(\frac{1}{Q_{2} k}\right)-\pi^{2} \delta\left(Q_{1} k\right) \delta\left(Q_{2} k\right)\right] \beta_{ \pm} \\
-\left[\mathbf{P}\left(\frac{1}{Q_{1} k}\right) \delta\left(Q_{2} k\right)+\mathbf{P}\left(\frac{1}{Q_{2} k}\right) \delta\left(Q_{1} k\right)\right] \alpha_{ \pm}
\end{gathered}
$$

where

$$
\lim _{\varepsilon \rightarrow 0} \frac{1}{D_{ \pm}\left(i k_{4} \rightarrow \omega+i \varepsilon\right)}=\alpha_{ \pm}+i \pi \beta_{ \pm}
$$

$\mathbf{P}$ denotes the principal part prescription. The detailed expressions for the functions $\alpha_{ \pm}$ and $\beta_{ \pm}$can be found in [13 - 17]. We show below that the mass singularities arise only when both $k$ and $k^{\prime}$ are space-like momenta.

\subsection{Mass singular contributions}

Taking the imaginary part of $\Pi_{\mu}^{\mu}$ leads to products of the type $\rho_{ \pm} \rho_{ \pm}^{\prime}, \sigma_{ \pm} \sigma_{ \pm}^{\prime}$ and $\tau_{ \pm} \tau_{ \pm}^{\prime}$ in eqs. $(7 \mathrm{a}),(7 \mathrm{~b})$ and $(7 \mathrm{c})$. The next step is to integrate over $d \Omega$, and over $d \Omega_{1}, d \Omega_{2}$, respectively.

Only the $\frac{1}{(Q k)}$ factors can produce singularities: e. g. in the product of terms $\sigma_{ \pm} \sigma_{ \pm}^{\prime}$ arising from eq. $(7 \mathrm{~b})$ we have 


$$
\mathbf{P}\left(\frac{1}{Q k}\right) \delta\left(Q k^{\prime}\right)=\mathbf{P}\left(\frac{1}{Q P}\right) \delta\left(Q k^{\prime}\right)=\frac{1}{(Q P)} \delta\left(Q k^{\prime}\right)
$$

(as $(Q P) \geq 0$ the $\mathbf{P}$ prescription is dropped); i.e. when $(Q P)=0$, i.e. $\widehat{Q} \rightarrow-\frac{\vec{p}}{E}$, $\left(Q^{2}=P^{2}=0\right)$, a non-integrable singularity appears. In the following we regularize this singularity by using dimensional regularisation of the angular integral over $d \Omega$ in $D=3+2 \widehat{\varepsilon}$ dimensions, with $\widehat{\varepsilon}>0$, but keeping only the singular parts :

$$
\left.\int \frac{d \Omega}{4 \pi} \rightarrow \int \frac{d \Omega}{4 \pi}\right|_{r e g} \stackrel{\text { def }}{=} \frac{1}{2} \int_{0}^{\pi} d \theta \sin ^{D-2} \theta=\frac{1}{2} \int_{-1}^{1} d \cos \theta\left(1-\cos ^{2} \theta\right)^{\widehat{\varepsilon}}
$$

By taking the discontinuity of eq. (7) using eq. (8), we thus retain only the products $\mathbf{P}\left(\frac{1}{Q k}\right) \delta\left(Q k^{\prime}\right)$ in order to compute the leading (singular) contribution to the soft photon production rate. The singularity arises when $(Q k)=\left(Q k^{\prime}\right)=0$, which is possible only for space-like $k$ and $k^{\prime}$. For this reason we restrict ourselves to this domain, since all other contributions are regular*.

In some more detail we describe the procedure for the term $\operatorname{tr}(1)$, eq. (7 b). The part giving rise to the singularity reads - after regularisation according to eq. (12) :

$$
\begin{gathered}
\left.\operatorname{Im} \Pi_{\mu}^{\mu}\right|_{1, r e g}=-\left.4 m_{f}^{2} \int[d k] \int \frac{d \Omega}{4 \pi}\right|_{r e g} \frac{1}{(Q P)} \\
\cdot\left\{\delta\left(Q k^{\prime}\right)\left[\beta_{+}(1+\widehat{k} \cdot \widehat{Q})+\beta_{-}(1-\widehat{k} \cdot \widehat{Q})\right]\left[\alpha_{+}^{\prime}\left(1+\widehat{k^{\prime}} \cdot \widehat{Q}\right)+\alpha_{-}^{\prime}\left(1-\widehat{k^{\prime}} \cdot \widehat{Q}\right)\right]\right. \\
\left.+\operatorname{sym}\left(k \longleftrightarrow k^{\prime}\right)\right\}
\end{gathered}
$$

where the continuation e.g. $(Q k) \Rightarrow \omega+\widehat{Q} \cdot \vec{k}$ is implied. $\alpha_{ \pm}, \beta_{ \pm}$are functions of $\omega$ and $k=|\vec{k}|$. The integrations with respect to $k, \omega, \omega^{\prime}$ are indicated by the short-hand notation:

$$
\int[d k] \equiv e^{2} e_{q}^{2} N_{c} \pi\left(1-e^{E / T}\right) \int \frac{d^{3} \vec{k}}{(2 \pi)^{3}} \int_{-|k|}^{|k|} d \omega \int_{-\left|k^{\prime}\right|}^{\left|k^{\prime}\right|} d \omega^{\prime} n_{F}(\omega) n_{F}\left(\omega^{\prime}\right) \delta\left(E-\omega-\omega^{\prime}\right) .
$$

In the limit $\left.\widehat{\varepsilon} \rightarrow 0 \operatorname{Im} \Pi_{\mu}^{\mu}\right|_{1, \text { reg }}$ behaves as $1 / \widehat{\varepsilon}$ : the residue is determined by replacing $\widehat{Q}$ by $-\frac{\vec{p}}{E}$. The integral over $d \Omega$ is then computed for $\widehat{\varepsilon} \rightarrow 0$ :

* No singularity is produced by the $\mathbf{P P}$ or $\delta \delta$ products present in the terms proportional to $\sigma_{ \pm} \sigma_{ \pm}^{\prime}$ and $\tau_{ \pm} \tau_{ \pm}^{\prime}$. 


$$
\left.\int \frac{d \Omega}{4 \pi}\right|_{r e g} \frac{1}{(Q P)} \simeq \frac{1}{2 E \widehat{\varepsilon}}
$$

The leading divergent behaviour expressed in terms of the factor $1 / \hat{\varepsilon}$ finally becomes :

$$
\begin{gathered}
\left.\operatorname{Im} \Pi_{\mu}^{\mu}\right|_{1, r e g}=-2 m_{f}^{2} \frac{1}{\widehat{\varepsilon}} \int[d k] \delta(P k) \\
\cdot\left\{\left(\beta_{+}\left(1-\frac{\omega}{k}\right)+\beta_{-}\left(1+\frac{\omega}{k}\right)\right)\left(\alpha_{+}^{\prime}\left(1-\frac{\omega^{\prime}}{k^{\prime}}\right)+\alpha_{-}^{\prime}\left(1+\frac{\omega^{\prime}}{k^{\prime}}\right)\right)\right. \\
\left.+\operatorname{sym}\left(\omega, k \longleftrightarrow \omega^{\prime}, k^{\prime}\right)\right\} .
\end{gathered}
$$

Next we discuss the contribution including two vertex corrections, i.e. the $\operatorname{tr}(2)$ term of eq. ( $7 \mathrm{c}$ ). Let us focus in the following on the " ++ " terms (the others being obtained by symmetry) and extract the potentially singular part from the $\tau_{+} \tau_{+}^{\prime}$ term. The expression of $\tau_{+}$(cf. eq. (10 c)) shows that terms in $\alpha_{+} \beta_{+}^{\prime}, \alpha_{+}^{\prime} \beta_{+}, \alpha_{+} \alpha_{+}^{\prime}$ and $\beta_{+} \beta_{+}^{\prime}$ will appear in the singular contribution, because each of the latter quantities can be associated with a $\mathbf{P}\left(\frac{1}{Q k}\right) \delta\left(Q k^{\prime}\right)$ product.

The contribution is :

$$
\begin{gathered}
\left.\operatorname{Im} \Pi_{\mu}^{\mu}\right|_{2, r e g,++}=\left.\left.2 m_{f}^{4} \int[d k] \int \frac{d \Omega_{1}}{4 \pi}\right|_{r e g} \int \frac{d \Omega_{2}}{4 \pi}\right|_{r e g}\left(Q_{1} Q_{2}\right) \\
\cdot\left[\left(Q_{1} k_{+}\right)\left(Q_{2} k_{+}^{\prime}\right)+\left(Q_{1} k_{+}^{\prime}\right)\left(Q_{2} k_{+}\right)-\left(Q_{1} Q_{2}\right)\left(k_{+} k_{+}^{\prime}\right)\right] \\
\cdot\left\{\frac{1}{\left(Q_{1} P\right)} \mathbf{P}\left(\frac{1}{Q_{2} k^{\prime}}\right) \mathbf{P}\left(\frac{1}{Q_{2} k}\right) \delta\left(Q_{1} k\right) \alpha_{+} \beta_{+}^{\prime}\right. \\
+\frac{1}{\left(Q_{1} P\right)} \mathbf{P}\left(\frac{1}{Q_{2} k}\right) \mathbf{P}\left(\frac{1}{Q_{2} k^{\prime}}\right) \delta\left(Q_{1} k^{\prime}\right) \alpha_{+}^{\prime} \beta_{+} \\
\left.-\frac{1}{\left(Q_{1} P\right)} \frac{1}{\left(Q_{2} P\right)}\left(\delta\left(Q_{1} k\right) \delta\left(Q_{2} k^{\prime}\right) \alpha_{+} \alpha_{+}^{\prime}-\pi^{2} \delta\left(Q_{1} k^{\prime}\right) \delta\left(Q_{2} k^{\prime}\right) \beta_{+} \beta_{+}^{\prime}\right)\right\}
\end{gathered}
$$

We find the $\frac{1}{\hat{\varepsilon}}$ contribution for $\widehat{Q_{1}} \rightarrow-\frac{\vec{p}}{E}$ or $\widehat{Q_{2}} \rightarrow-\frac{\vec{p}}{E}$ (the latter is obtained by symmetry $k \leftrightarrow k^{\prime}$ ). No double pole has to be considered because of the presence of the $\left(Q_{1} Q_{2}\right)$ factor.

Using :

$$
\int \frac{d \Omega}{4 \pi} \delta(Q k)=\frac{\theta\left(-k^{2}\right)}{2|\vec{k}|}
$$




$$
\int \frac{d \Omega}{4 \pi} \delta\left(Q k^{\prime}\right)(Q k)=\frac{\theta\left(-k^{2}\right)}{2\left|\vec{k}^{\prime}\right|^{2}}\left(\omega\left|\vec{k}^{\prime}\right|-\omega^{\prime} \vec{k} \cdot \widehat{k^{\prime}}\right)
$$

and $[11]$ :

$$
\begin{gathered}
\int \frac{d \Omega}{4 \pi} \mathbf{P}\left(\frac{1}{k Q}\right)=L(k) \equiv L=\frac{1}{2 k} \ln \left|\frac{\omega+k}{\omega-k}\right|, \\
\int \frac{d \Omega}{4 \pi} \mathbf{P}\left(\frac{k^{\prime} Q}{k Q}\right)=\omega^{\prime} L-\frac{\vec{k} \cdot \vec{k}^{\prime}}{\vec{k}^{2}}(\omega L-1)
\end{gathered}
$$

we obtain after combining the contributing terms :

$$
\begin{gathered}
\left.\operatorname{Im} \Pi_{\mu}^{\mu}\right|_{2, \text { reg },++}=m_{f}^{4} \frac{1}{\widehat{\varepsilon}} \int[d k] \frac{\delta(P k)}{E} \\
\cdot\left\{\left(\alpha_{+} \beta_{+}^{\prime}+\alpha_{+}^{\prime} \beta_{+}\right)\left[2(1-\omega / k)\left(1-\omega^{\prime} / k^{\prime}\right) L+\left((E-k) / k^{\prime}-1\right)\left(\left(1-\frac{\omega^{2}}{\vec{k}^{2}}\right) L+\frac{\omega}{\vec{k}^{2}}\right)\right]\right. \\
+\left(-\alpha_{+} \alpha_{+}^{\prime}+\pi^{2} \beta_{+} \beta_{+}^{\prime}\right) \frac{1}{2 k}\left[2(1-\omega / k)\left(1-\omega^{\prime} / k^{\prime}\right)+\left(1-\frac{\omega^{2}}{k^{2}}\right)\left((E-k) / k^{\prime}-1\right)\right] \\
\left.+\operatorname{sym}\left(\omega, k \longleftrightarrow \omega^{\prime}, k^{\prime}\right)\right\} .
\end{gathered}
$$

Similar contributions come from $\tau_{+} \tau_{-}^{\prime}$ and $\tau_{-} \tau_{-}^{\prime}$, respectively.

The functions $L$ (and $L^{\prime}$ ) in eq. (20) are eliminated by using the definitions for $\alpha_{ \pm}$and $\beta_{ \pm}$, eq. (10 d) :

$$
\begin{gathered}
m_{f}^{2}(1-\omega / k) L \alpha_{+}=\left(\omega-k-\frac{m_{f}^{2}}{k}\right) \alpha_{+}-\pi^{2} \frac{m_{f}^{2}}{2 k}(1-\omega / k) \beta_{+}+1, \\
m_{f}^{2}(1-\omega / k) L \beta_{+}=\left(\omega-k-\frac{m_{f}^{2}}{k}\right) \beta_{+}+\frac{m_{f}^{2}}{2 k}(1-\omega / k) \alpha_{+} .
\end{gathered}
$$

Thus eq. (20) contains terms proportional to $\alpha_{+} \alpha_{+}^{\prime}, \alpha_{+} \beta_{+}^{\prime}, \alpha_{+}^{\prime} \beta_{+}$and $\beta_{+} \beta_{+}^{\prime}$, and terms linear in $\beta_{+}, \beta_{+}^{\prime}$. The $\alpha_{+} \alpha_{+}^{\prime}$ and $\beta_{+} \beta_{+}^{\prime}$ terms vanish in eq. (20), whereas the $\alpha_{+} \beta_{+}^{\prime}$ and $\alpha_{+}^{\prime} \beta_{+}$terms compensate with those of eq. (16). All what remains are the linear terms in $\beta_{+}$and $\beta_{+}^{\prime}$. The final result (including all contributions from $\tau_{ \pm} \tau_{ \pm}^{\prime}$ ) is :

$$
\left.\operatorname{Im} \Pi_{\mu}^{\mu}\right|_{r e g}=2 m_{f}^{2} \frac{1}{\widehat{\varepsilon}} \int[d k] \frac{\delta(P k)}{E}
$$




$$
\text { - }\left\{\beta_{+}^{\prime}\left(1-\omega^{\prime} / k^{\prime}\right)+\beta_{+}(1-\omega / k)+\beta_{-}^{\prime}\left(1+\frac{\omega^{\prime}}{k^{\prime}}\right)+\beta_{-}\left(1+\frac{\omega}{k}\right)\right\} \text {. }
$$

This expression may still be simplified by following a procedure familiar from the hard photon case [6]. Since the functions $\beta_{+}$and $\beta_{-}$as given by [13-17] are peaked for $\omega \rightarrow 0$ ( $k$ being fixed) we may replace $n_{F}(\omega) n_{F}(E-\omega)$ by $n_{F}(0) n_{F}(E)=\frac{1}{2} n_{F}(E)$. After performing the angular integration in eq. (22), the remaining integrals $\int d \omega\left(1 \mp \frac{\omega}{k}\right) \beta_{ \pm}(\omega, k)$ are evaluated using the sum rule [6] :

$$
\int_{-\infty}^{\infty} d \omega(1 \mp \omega / k) \rho_{ \pm}(\omega, k)=0
$$

The dominant contribution of the integral over $k$ comes from $m_{f}<k<T$. The leading contribution for $g \rightarrow 0$ then reads :

$$
E \frac{d W}{d^{3} \vec{p}} \simeq \frac{1}{\widehat{\varepsilon}} \frac{e_{q}^{2} \alpha \alpha_{s}}{2 \pi^{2}} T^{2} n_{F}(E)\left(\frac{m_{f}}{E}\right)^{2} \ln \left(\frac{1}{\alpha_{s}}\right)
$$

This result shows that the Braaten-Pisarski resummation does not yield a finite soft real photon production rate: a logarithmic divergence remains.

\section{DISCUSSION}

The above analysis allows to identify the diagrams which are responsible for the singularities as they originate from terms proportional to the product $\mathbf{P}\left(\frac{1}{Q k}\right) \delta\left(Q k^{\prime}\right)$. One example of such a diagram is shown in Fig. 2, where the singularity is due to the massless quark exchange present in the hard thermal loop effective vertices. The massless exchange is transparent in the two $\rightarrow$ three amplitude of Fig. 2b. The singularity arises from the configuration $Q \cdot P=0$ : it corresponds to a collinear singularity when the photon is allowed to stay massless, $P^{2}=0$.

At present we do not know how to screen this mass singularity by a consistent prescription. Therefore a pragmatic approach is to introduce a soft cut-off of $O(g T)$ in order to regularize the soft photon production rate at logarithmic accuracy :

$$
E \frac{d W}{d^{3} \vec{p}} \simeq e_{q}^{2} \alpha \alpha_{s} T^{2} \ell n^{2}\left(\frac{1}{\alpha_{s}}\right)
$$

where the photon energy is assumed $E \sim m_{f}$. 
The presented result is valid for soft massless, i.e. non-thermalized photons. This implies that the quark - gluon plasma has to have a finite size; its characteristic length is denoted by $L$. As already mentioned in the Introduction the mean free path $l_{\gamma}$ of the detected photon has to be larger than $L$. Since only photons with wave lengths less than $L$ are radiated, the dimension of the plasma becomes constrained [18] :

$$
\frac{2 \pi}{E}<L<l_{\gamma}(E) .
$$

Suppressing the logarithmic factors in eq. (25) we estimate the photon's mean free path to be given by :

$$
l_{\gamma} \simeq \frac{E}{\alpha \alpha_{s} T^{2}} .
$$

This order of magnitude estimate is in agreement with $l_{\gamma} \simeq 1 / n_{q} \sigma_{\text {Compton }}(E)$, where $n_{q}$ is the quark density and $\sigma_{\text {Compton }}(E) \sim \alpha \alpha_{s} / E T$ is the high energy Compton cross section in the QGP, which is responsible for the photon absorption.

For soft photon energies $E \sim m_{f} \sim O(g T)$ the constraint eq. (26) becomes:

$$
O(1)<L T \sqrt{\alpha_{s}}<O\left(\frac{1}{\alpha}\right)
$$

i.e. for typical values of $T \sim 400 \mathrm{MeV}$ and $\alpha_{s} \sim 0.25$ the constraint reads : $1 \mathrm{fm}<L<$ $100 \mathrm{fm}$. This size is compatible with the expectations for a realistic QGP produced in heavy - ion collisions.

In summary it seems reasonable to forsee experimental situations where soft $O(g T)$ non-thermalized photons would be emitted from a QGP. However, our present understanding does not allow us to derive their finite production rate. 


\section{ACKNOWLEDGEMENTS}

We kindly thank P. Aurenche, J. Kapusta and R. D. Pisarski for helpful remarks, and E. Pilon for discussions. Partial support of this work by "Projets de Coopération et d'Echange" (PROCOPE) is gratefully acknowledged.

\section{REFERENCES}

1) For a recent review: P. V. Ruuskanen, in Particle Production in Highly Excited Matter, eds. H. H. Gutbrod and J. Rafelski, Proc. of ASI, Il Ciocco, Lucca (Italy), 1993

2) K. Kajantie and H. I. Miettinen, Z. Phys. C9 (1981) 341; and earlier references quoted therein.

3) K. Kajantie and P. V. Ruuskanen, Phys. Lett. B121 (1983) 352.

4) M. Neubert, Z. Phys. C - Particles and Fields C42 (1989) 231.

5) J. I. Kapusta, P. Lichard, and D. Seibert, Phys. Rev. D44 (1991) 2774.

6) R. Baier, H. Nakkagawa, A. Niégawa, and K. Redlich, Z. Phys. C - Particles and Fields C53 (1992) 433.

7) E. Shuryak and L. Xiong, Phys. Rev. Lett. 70 (1993) 2241; A. Makhlin, preprint SUNY-NTG-93-10, January 1993.

8) For a review and references: R. D. Pisarski, Nucl. Phys. A525 (1991) 175c; and E. Braaten, Nucl. Phys.(Proc. Suppl.) B23 (1991) 351.

9) R. D. Pisarski, Nucl. Phys. B309 (1988) 476; Phys. Rev. Lett. 63 (1989) 1129.

10) E. Braaten and R. D. Pisarski, Phys. Rev. Lett. 64 (1989) 1338; Nucl. Phys. B337 (1990) 569; Nucl. Phys. B339 (1990) 310.

11) J. Frenkel and J. C. Taylor, Nucl. Phys. B334 (1990) 199.

12) T. Altherr and P. V. Ruuskanen, Nucl. Phys. B380 (1992) 377.

13) E. Braaten, R. D. Pisarski, and T. C. Yuan, Phys. Rev. Lett. 64 (1990) 2242;

14) S. M. H. Wong, Z. Phys. C - Particles and Fields C53 (1992) 465.

15) V. V. Klimov, Sov. J. Nucl. Phys. 33 (1981) 934; O. K. Kalashnikov, Fortschr. Phys. 32 (1984) 525.

16) H. A. Weldon, Phys. Rev. D26 (1982) 2789; Physica A158 (1989) 169; and Phys. Rev. D40 (1989) 2410.

17) R. D. Pisarski, Physica A158 (1989) 146; Fermilab preprint Pub - 88/113-T (unpublished).

18) For a discussion of soft photon radiation in a QED plasma: H. A. Weldon, Phys. Rev. D44 (1991) 3955. 


\section{FIGURE CAPTIONS}

Fig.1: One-loop diagram for the production of a real soft photon (weavy line) with momentum $P$. The effective quark propagator and the effective quark-photon vertex are indicated by a blob.

Fig.2: (a) Cutting the effective one-loop diagram through the effective hard thermal loop vertex gives rise to (b) the amplitude with a collinear singularity for $P \cdot Q=0$. The curly line denotes the gluon. 
This figure "fig1-1.png" is available in "png" format from: http://arxiv.org/ps/hep-ph/9311329v1 